\title{
Analysis of selected geometrical features of a structural element on the example of a dental implant
}

\author{
Szymon Imiłkowski ${ }^{1}$, Stepan Kovalyshyn ${ }^{2}$, Karolina Karolewska ${ }^{1}$, Bogdan Ligaj $^{1}$ and \\ Mateusz W irwicki1, \\ ${ }^{1}$ UTP University of Sciences and Technology in Bydgoszcz, Faculty of M echanical Engineering AI. \\ Prof. S. Kaliskiego 7; 85- 796, Bydgoszcz; Poland \\ ${ }^{2} \mathrm{~L}$ viv National A gricultural University, Faculty of $M$ echanization and Power Engineering, street $\mathrm{V}$. the \\ Great, 1, Zhovkva district, L viv region, 80381 Dublyany, Ukraine
}

\begin{abstract}
The aim of the article was to carry out FEM strength tests for four proposed by the authors geometry of the intraoral implant. Change of their geometrical features to find the most optimal distribution of reduced stresses. The article presents various geometries of the new dental implant. Strength tests were performed using the finite element method (FEM). The results of the tests were analyzed and their assessment was made for which of the solutions of the new geometry the stress distribution is the most optimal. Detailed results of the analyzes for a given model are shown and new solutions in the construction of the implant are proposed.
\end{abstract}

\section{Introduction}

Threaded connections used in typical fasteners made of classic construction materials require strength calculations [1]. In such cases, threaded connections with a metric profile are able to provide a favorable stress pattern in the joined elements. In the case of titanium implant connections with bone, due to the disproportion of the mechanical properties of the materials that are in contact (titanium-bone), the use of a metric thread in the implant leads to a very unfavorable stress distribution, in particular in the cancellous bone, because it is quite a delicate bone and already it could be damaged under a relatively small load [2]. In implantology, for example, I use a double thread, they are the same threading and self-cutting the bone, it allows you to achieve some primary stabilization even in the case of soft bone. Another variant is the open thread, which prevents necrosis and has the unique ability to sink into the bone and allows for the correction of the depth of implant placement during the procedure, it also has the option of changing the axis of implantation immediately after the procedure [3]. The conical connection is able to ensure tightness. The connection by means of a Morse cone with an indexing hex definitely facilitates the assembly of prosthetic works [4]. The strength of the material used for the production of intra-bone implants can be increased by applying appropriate heat treatment, e.g. annealing [5, 6]. The conducted experimental tests on a testing machine with the use of optical cameras recording the course of material destruction allow for the development of more durable structural elements intraoral implants $[7,8]$. During the design work, attention must be paid to the manufacture of all-ceramic crowns and the treatment of their surfaces [9]. These materials must be tested for aging in the aquatic environment and gradual degradation of the material and structure [10]. FEM tests can be used in various industries for quick and non-destructive determination of strength parameters of entire structures or individual elements [11].

\footnotetext{
* Corresponding author: wirwicki@utp.edu.pl
} 
The aim of the article is to carry out FEM strength tests for four proposed by the authors geometry of the intraoral implant. Change of their geometrical features to find the most optimal distribution of reduced stresses.

\section{Materials and Methods}

\subsection{Geometry of a construction element}

The study used a jaw that was scanned with a 3D Ceramill MAP 300 dental scanner and exported to the Autodesk Inventor Professional 2020 environment. Model of the lower jaw has been simplified. The next step is to design the new geometry of the dental implant, which is shown in Figure 1.

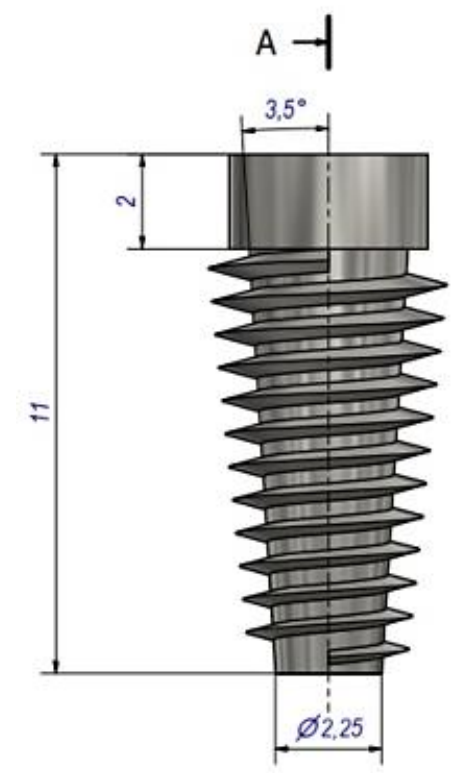

$A \rightarrow 1$

$B(20: 1)$

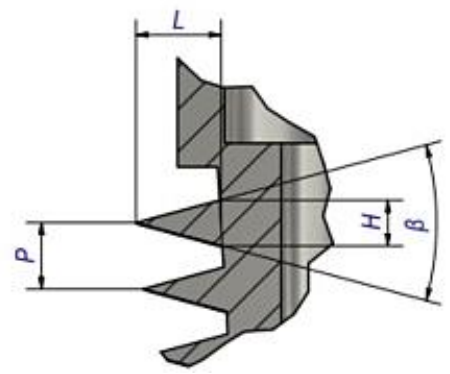

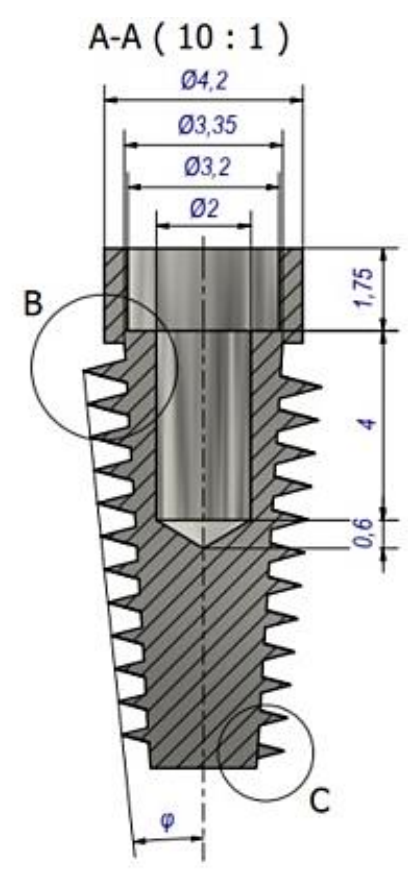

$C(20: 1)$

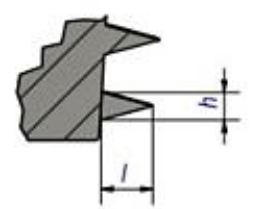

Fig. 1. The geometry of the new dental implant used for research.

Table 1 presents detailed parameters of the new implant geometry, where: L - width of the first thread turn, 1 - width of the last thread turn, $\mathrm{H}$ - height of the first thread turn, $\mathrm{h}$ - height of the last thread turn, $\mathrm{P}$ - thread pitch, $\varphi$ - angle of the thread turns, $\beta$ - thread cross-section angle. 
Table 1. Parameters of the new implant geometry.

\begin{tabular}{|c|c|c|c|c|}
\hline Geometry & $\mathbf{1}$ & $\mathbf{2}$ & $\mathbf{3}$ & $\mathbf{4}$ \\
\hline $\mathbf{L}(\mathbf{m m})$ & 0,9 & 0,9 & 0,9 & 0,9 \\
\hline $\mathbf{I}(\mathbf{m m})$ & 0,55 & 0,1 & 0,55 & 0,11 \\
\hline $\mathbf{H}(\mathbf{m m})$ & 0,48 & 0,48 & 0,66 & 0,63 \\
\hline $\mathbf{h}(\mathbf{m m})$ & 0,29 & 0,05 & 0,4 & 0,08 \\
\hline $\mathbf{P}(\mathbf{m m})$ & 0,7 & 0,7 & 0,7 & 0,7 \\
\hline $\boldsymbol{\varphi (})$ & 6 & 9 & 6 & 9 \\
\hline $\boldsymbol{\beta}(\boldsymbol{)})$ & 30 & 30 & 40 & 40 \\
\hline
\end{tabular}

\subsection{Loading conditions}

The implant was pressurized in two places. The stresses were defined as follows: for the "pressure 1" symbol, the surface area is $5.812 \mathrm{~mm} 2$ and the force with which we interact is $50 \mathrm{~N}$. For the first and second pressure, the force is the same as in total, it makes the load force $100 \mathrm{~N}$, which is evenly distributed over two areas of the implant surface to which the crown will be attached. The load applied to the implant acts axially. The stress was calculated from Pascal's formula and is as follows for "pressure 1" it is $8.602 \mathrm{MPa}$ and for "pressure 2" it is $10.2 \mathrm{MPa}$. The area for "pressure $2 "$ is $4.901 \mathrm{~mm} 2$. Figure 2 shows the places where the pressure is applied.

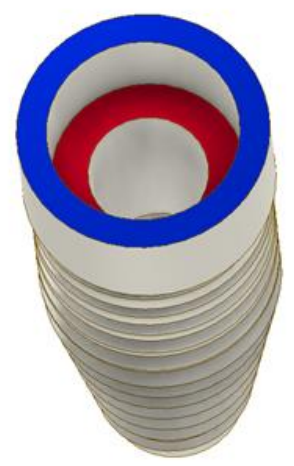

Fig. 2. New implant geometry: pressure 1 - blue, pressure 2 - red.

The implant was placed in a previously prepared lower jaw. For the purposes of the research, the mandible model has been simplified and the material parameters presented in Table 2 have been assigned.

Table 2. Material parameters for bones.

\begin{tabular}{|c|c|c|}
\hline Material & Young modulus, GPa & Poisson's ratio \\
\hline Compact tissue & 1,5 & 0.3 \\
\hline Trabecular bone & 0.15 & 0.3 \\
\hline
\end{tabular}

Figure 3 shows the model used to perform the strength analysis using the finite element method (FEM) in the Ansys program. 


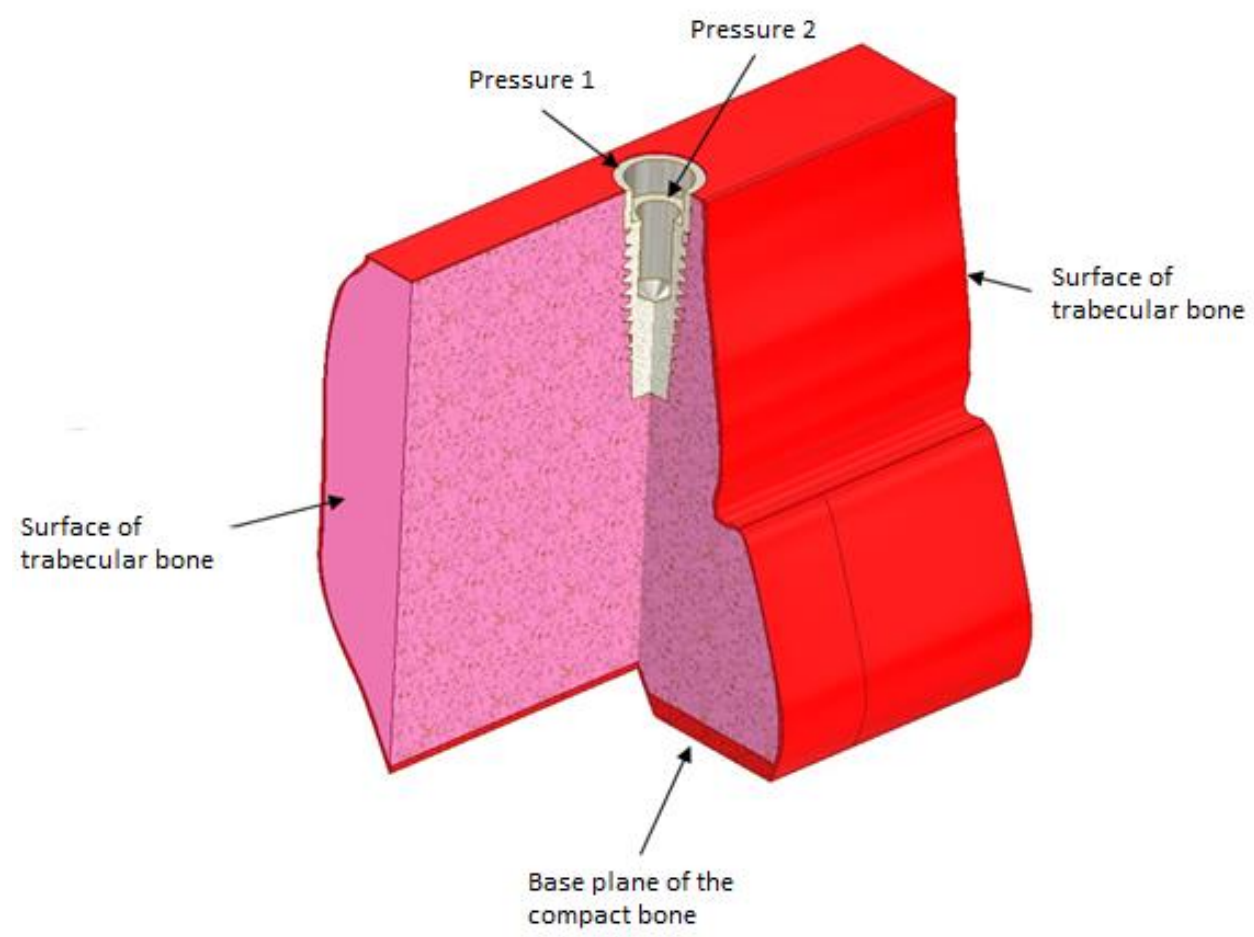

Fig. 3. A model of a mandibular dissection with a new implant geometry used for FEM tests.

The plane of the base is modeled to reflect the jaw, and where it begins to expand, it is simplified and fixed. The bone length was set at $30 \mathrm{~mm}$ and the implant was placed in the middle. The lower jaw is drawn from the model of a scanned human lower jaw. The simplification of the model is necessary to perform the research. After preliminary calculations of the mandible, the assumed length of $30 \mathrm{~mm}$ turned out to be the most optimal. The tooth roots next to our implant were not included in the research.

\section{Results and Analysis}

The research was carried out on four models of implants placed in the same conditions and loaded in the same way. For this type of research, the finite element mesh should be modified appropriately to improve its parameters by removing strongly degenerate elements. The analysis focused on the stresses on the core and on the implant thread located in the compact and spongy bone in the maxilla. They will be assessed on each model separately, in which the implant model has been isolated, to facilitate the assessment and recognition of stresses in a given example. The stresses acting in the overall model will be checked and the displacement of the jaw bone along with the inserted implant will be analyzed. Figure 4 shows the reduced stresses occurring in the implant. 
a)

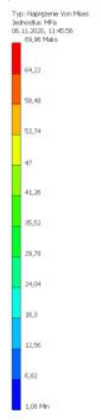

c)

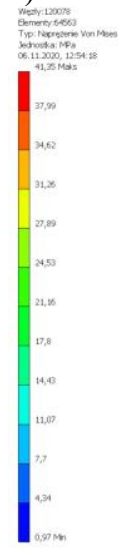

b)
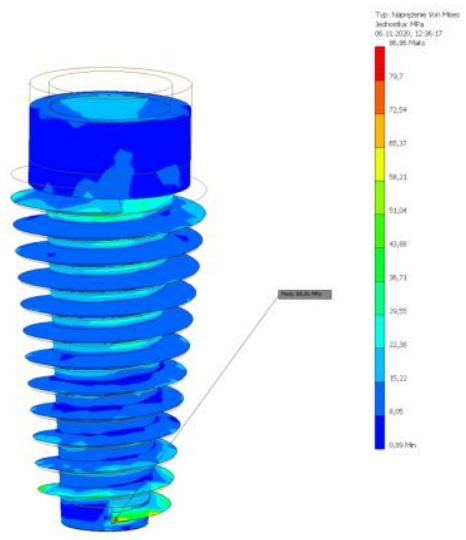

d)

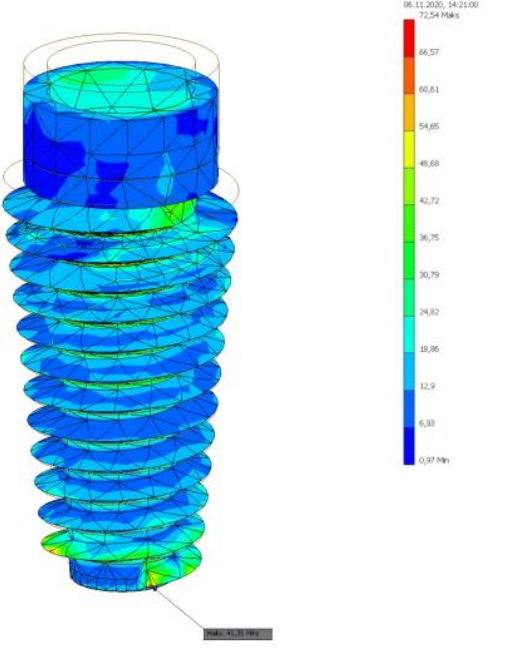

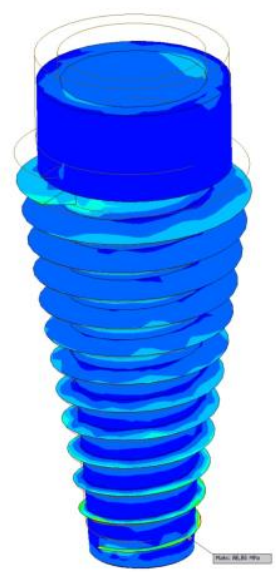

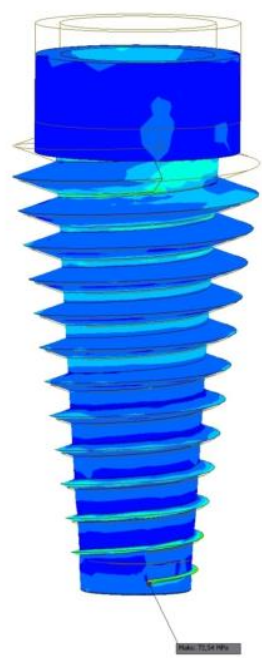

Fig. 4. New implant geometry with a stress map: a) geometry 1, b) geometry 2, c) geometry 3, d) geometry 4.

Geometry 1 has a 6 degree tapered thread where the core is 3.5 degrees. The greatest stress occurs at the end of the last thread turn and is almost 70MPa. Such a high stress is caused by the size of this element and its location and the force applied axially. The stress of the implant core is in the range of $1.08-29.78 \mathrm{MPa}$, however, most of it is in the range of $6.82-18.3 \mathrm{MPa}$. In geometry 2 , the stress distribution shows that the most loaded element is the thread, and exactly 3 threads from the bottom, the last one, similarly to the previous example, transfers the greatest stress and the first thread within the range from 15.22 to 29.55 $\mathrm{MPa}$. The implant core, as we can see, is fairly evenly loaded and the stress mostly ranges from 0.89 to $22.38 \mathrm{MPa}$. In geometry 3 we notice a different stress distribution than in the above. The highest stress occurs at the end of the coil and is $41.35 \mathrm{MPa}$. The last two turns, together with the first two, are heavier than the rest. The implant core is particularly heavily loaded than in the other examples. This is due to a 40-degree thread angle. In the last geometry, a greater number of loaded turns is noticeable than in the previous geometries. The highest stress of $72.54 \mathrm{MPa}$ occurs at the end of the thread turn. The first turn is not loaded in a similar way to the lower one, where almost 4 turns are visibly loaded. The core in the 
implant is loaded more at the top, representing it with top-bottom stress values, it is as follows: $30.79-0.97 \mathrm{MPa}$.

The next element of the research was the analysis of stress areas occurring in the new geometry of the maxillary implant. Figure 5 shows the areas subject to stress analysis.

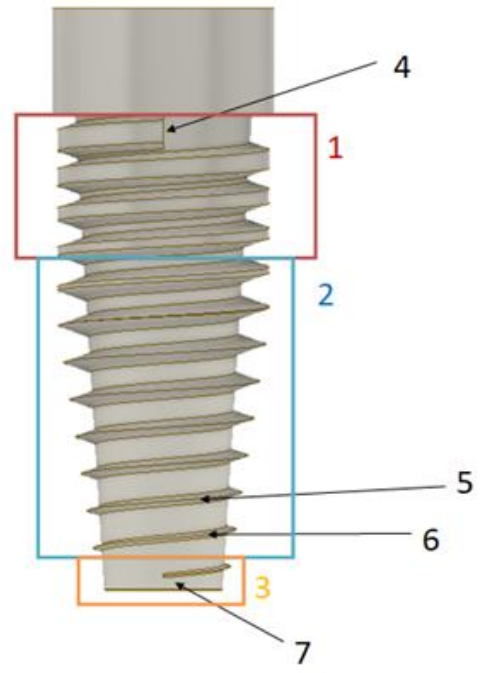

Fig. 5. Stress analysis areas in thread geometry.

The number 1 indicates the area in which the tapered area of the thread core will be assessed. Number 2 will be used to evaluate the implant core in the marked place. Number 3 will be used to assess the stress state at the end of the thread core, however the area marked with number 3 will represent the highest stress value. The thread will be assessed on the individual turns, and so on the first turn it is number 4, on the 10th turn number 5 and successively 11 th turn number 6 . The number 8 will indicate the greatest stress occurring in the model. The table below shows the stress values for the model and designated areas of the new geometry of the maxillary implant.

Table 3. Stress values in the studied areas of implant models, $M$ pa.

\begin{tabular}{|c|c|c|c|c|}
\hline & Model 1 & Model 2 & Model 3 & Model 4 \\
\hline 1 & $12,6-41,3$ & $15,2-36,7$ & $14,4-27,9$ & $6,9-30,8$ \\
\hline 2 & $6,8-18,3$ & $0,9-22,4$ & $4,34-27,9$ & $1-18,9$ \\
\hline 3 & 12,6 & 15,2 & 11,1 & 6,9 \\
\hline 4 & $6,8-29,8$ & $8,1-36,7$ & $4,3-27,9$ & $1-18,9$ \\
\hline 5 & $6,8-29,8$ & $8,1-36,7$ & $4,3-21,2$ & $6,9-30,8$ \\
\hline 6 & $6,8-41,3$ & $8,1-36,7$ & $0,97-17,9$ & $6,9-36,8$ \\
\hline 7 & $12,6-64,2$ & $22,4-86,9$ & $4,3-38$ & $6,9-42,7$ \\
\hline 8 & 70 & 86,9 & 41,4 & 72,5 \\
\hline
\end{tabular}

The stresses occurring in the presented geometries were caused by axial pressure. Four models were proposed for the analysis. A solution that is satisfactory in terms of geometry is one that when we load the implant axially, the stress is not only transmitted through the thread, but also through the core. The research carried out on the model is to guide the further development of implant geometry in order to be able to design the best products. However, the presented values clearly show what is happening in the mandible in which the implant 
with the given geometry will be implemented. The thread pitch in the models is the same, which means that it does not affect the obtained results, as does the angle of the core taper. The variable elements were the thread contour and the coil angle as well as the thread angle marked in the implant structure as the $\beta$ angle. When analyzing the thread angle, where two 6 and 9 degrees have been analyzed, the 6 degrees angle is more favorable and thanks to it, more optimal stresses are obtained throughout the implant. Models 1 and 3 have an angle of 6 degrees, thanks to which the last thread protrudes more from the core cone and thus is able to hold the implant in the bone more tightly and each subsequent thread comes out more and more from the core. The stresses occurring in the implant core are most preferably presented in the upper part when the thread profile angle is 6 degrees, and the thread angle is 40 degrees, marked in the drawing with variables as $\beta$. The part marked with point 2 in the illustration shows the location of the core between the upper and lower segments, in which the thread profile angle of 6 degrees is the best. The angle adopted for the tests was not smaller due to the design features of the implant, which define that the thread is to hide in the part of the core going downwards, thanks to which its location in the bone is easier and desirable. The bottom of the core in models with a thread angle of 6 degrees is similar, and where there is an angle of 9 degrees, there is a significant discrepancy, which may be due to the fact that the angle marked as $\beta$ varies and when the angle is 30 degrees the thread is not able to " keep the implant in the bone and transfer the greatest stress to its base, and when this angle is 40 degrees, it is able to stick to the bone best, which results in the lowest value at this point. The first turn is the least loaded in model 4, and in model 2, the difference between these models is the $\beta$ angle. When assessing the lower thread turns, and at the same time the first threads that are screwed in, the model 3 presented the most advantageously. The end element of the thread can transfer the highest stresses due to its size and the size of the applied mesh. As can be seen from the results, the selection of the appropriate angle of the implant core as well as the angle $\varphi$ and the angle $\beta$ is necessary to create a very good implant. However, we must remember about the design features of the implant, and not only about creating a mechanically very durable product, because the strength properties of titanium in comparison with the strength of the dense and spongy bone, i.e. the socket where the implant model is to be mounted, differ significantly. The stresses arising in the implant are transferred to the mandible. In this work, the focus was only on the task of an axial load of $100 \mathrm{~N}$ and the results only present such assumptions.

\section{Conclusion}

The study discusses the strength of an implant made of titanium and placed in the jaw, which was subjected to strength tests. An anatomical orofacial model is shown to illustrate to the reader the complex structure of these elements of the human body. A part of the mandible consisting of two compact and spongy bones was accepted for research. The bones were scanned and the model was then mapped for strength analyzes. The research consisted in checking the effect of axial load on the jaw bone carried by the implant. The analysis was performed on four implant models with different geometries. It can be seen from the results that for a given implant whose core is at an angle of $3.5^{\circ}$, a thread coil placed at an angle of $6^{\circ}$ transfers loads more optimally than a coil with an angle of $9^{\circ}$. The angle in the thread marked as $\beta$, the greater it is, the smaller the stress result is on the thread line and greater on the core. However, it should be remembered that the test object was loaded axially and with such a force, the aim was to make the core take the load, because, as we know, the implant affects the mandibular bone. The environment in which it is placed, along with the geometry they present, can be subjected to many strength analyzes to search for the most appropriate and optimal structure of this element. The model calculated by its structure shows that there are many parameters that we are able to change in order to obtain the best possible stress distribution in the bone. 


\section{References}

1. F.J.de Cos Juez, F.Sánchez Lasheras, P.J.García Nieto, A Álvarez-A renal, Non-linear numerical analysis of a double-threaded titanium alloy dental implant by FEM, Appl. M ath. and Comp. Vol. 206, 2, pp. 952-967, (2008)

2. O. Cantó-Navés, X. Marimon, M. Ferrer, J. Cabratosa-Termesa, Comparison between experimental digital image processing and numerical methods for stress analysis in dental implants with different restorative materials, Journal of the M echanical B ehavior of Biomedical M aterials, V ol. 113, (2021)

3. O. Kayabasi, Design methodology for dental implant using approximate solution techniques, J ournal of Stomatology, Oral and M axillofacial Surgery, Available online, 22 January 2020

4. M.Shash, H.Nazha, W.Abbas, Influence of Different Abutment Designs on the Biomechanical B ehavior of One-Piece Zirconia Dental Implants and Their Surrounding Bone: A 3D-FEA, IRBM , V ol 40, 6, pp. 313 - 319, (2019)

5. M. W irwicki, Effect of the aging process at a temperature of 300 degrees Celsius of Ti6Al4V alloy on mechanical strength under static loading conditions, 9th International Conference On M anufacturing Science And Education (M se 2019): Trends In New Industrial Revolution, 5- 7 June, Sibiu, Romania, (2019)

6. K.K arolewska, B. Ligaj, M. W irwicki, G. Szala, Strength analysis of Ti6A I4V titanium alloy produced by the use of additive manufacturing method under static load conditions, J M R \& T, V ol. 9, 2 pp. 1365-1379, (2020)

7. K. Karolewska, B. Ligaj, Mechanical properties comparison of Ti6Al4V produced by different technologies under static load conditions, 9th International Conference On Manufacturing Science And Education (M se 2019): Trends In New Industrial Revolution, 5- 7 June, Sibiu, Romania, (2019)

8. K. Karolewska, B. Ligaj, D. Boroński, Strain Analysis of Ti6A I4V Titanium Alloy Samples U sing Digital Image Correlation, M aterials, V ol 13, 15, (2020)

9. M. Wirwicki, Analysis of the impact of the machining method and material environmental conditions on the strength of ZrO2, J M R \& T, V ol. 9, 3 pp. 2645-2651

10. A. Andrzejewska, M. Wirwicki, A. Andryszczyk, P. Siemianowski, Procedure for Determining Aqueous Medium Absorption in Biopolymers, 3rd International M aterials, Industrial And M anufacturing Engineering Conference (M imec2017), A IP Conference Proceedings, $\mathrm{V}$ ol 1902, (2017)

11. B. Ligaj, M. Wirwicki, K. Karolewska, A. Jasinska, Experimental verification of numerical calculations of railway passenger seats, 3rd International Conference On Science, Technology, A nd Interdisciplinary Research (IC-Star), IOP Conference Series$M$ aterials Science and Engineering, $\mathrm{V}$ ol 344, (2018) 